\title{
Mitochondrial Localization and Ocular Expression of Mutant Opa3 in a Mouse Model of 3-Methylglutaconicaciduria Type III
}

\author{
Kate A. Powell, ${ }^{1}$ Jennifer R. Davies, ${ }^{1}$ Elaine Taylor, ${ }^{1}$ Michael A. Wride, ${ }^{2}$ \\ and Marcela Votruba ${ }^{1,3}$
}

Purpose. To investigate the developmental and ocular expression of Opa3 in a mouse model of 3-methylglutaconicaciduria type III and the effect of mutation on protein localization and mitochondrial morphology.

Methods. The B6 C3-Opa3 ${ }^{\mathrm{L} 122 \mathrm{P}}$ mouse carrying a missense mutation in exon 2 (c.365T $>$ C; p.L122P) of Opa3, which displays features of recessive 3-methylglutaconic aciduria type III was studied. The expression of Opa3 was determined with RT-PCR, quantitative PCR, and Western blot, in embryos (embryonic day [E]8 to postnatal day [P]0) and adult tissues, and by ocular immunohistochemistry. Mitochondria were stained using a mitochondrion-selective probe in mouse embryonic fibroblasts from $\mathrm{Opa} 3^{-1-}$ mutants and imaged by electron microscopy of the retinas.

Results. The splice variants Opa3a and Opa3b were expressed in the lenses and the retinas in the $\mathrm{Opa} 3^{-/-}$mice, with the expression of the Opa3a isoform predominant. Opa3 was expressed throughout embryonic development, with high levels of expression in the developing brain, retina, optic nerve, and lens. Opa3 localized to the mitochondria, and the L122P mutant protein did not mislocalize Neither protein localized to the peroxisome. Opa3 ${ }^{-/-}$mice displayed disrupted mitochondrial morphology in the retina. Wild-type Opa3 protein increased as the lenses aged, despite the reduction in Opa3 mRNA occurring as a part of lens differentiation. However, mutant Opa3 mRNA was upregulated in homozygous mutant lenses, suggesting a compensatory increase in expression, which may further increase Opa3 protein levels.

Conclusions. Mutant Opa3 protein retains its mitochondrial localization and induces disrupted mitochondrial morphology. Opa3 accumulates in the lens. The results may reflect a slow turnover of Opa3 protein in vivo and may be important in normal lens physiology. (Invest Ophthalmol Vis Sci. 2011;52: 4369 - 4380) DOI:10.1167/iovs.10-6406

From the ${ }^{1}$ School of Optometry and Vision Sciences, Cardiff University, Cardiff, Wales, United Kingdom; the ${ }^{2}$ Ocular Developmental and Neurobiology Research Group, Zoology Department, School of Natural Sciences, University of Dublin, Trinity College, Dublin, Ireland; and the ${ }^{3}$ Cardiff Eye Unit, University Hospital Wales, Cardiff, Wales, United Kingdom.

Supported by the Medical Research Council Grant G0500790.

Submitted for publication August 13, 2010; revised December 14, 2010, and April 4, 2011; accepted April 18, 2011.

Disclosure: K.A. Powell, None; J.R. Davies, None; E. Taylor, None; M.A. Wride, None; $M$. Votruba, None

Corresponding author: Marcela Votruba Cardiff School of Optometry and Institute of Vision, Maindy Road, Cardiff CF24 4LU, UK votrubam@cardiff.ac.uk.
$\mathrm{H}$ ereditary optic neuropathies are a group of heterogeneous disorders in which degeneration of the retinal ganglion cells and optic nerve atrophy are the primary characteristics. Autosomal dominant optic atrophy (ADOA; MIM [Mendelian Inheritance in Man] 165500) is the most common of these disorders and is frequently associated with mutation of the OPA1 gene. ${ }^{1,2}$ However, optic atrophy has also been linked to mutation in an unrelated nuclear gene, OPA3. $O P A 3$ is associated with two phenotypically distinct conditions. ADOA and cataract are characterized by optic atrophy, extrapyramidal signs, and blue-dot cerulean cataract (ADOAC, MIM $165300^{3,4}$ ). Two dominant missense mutations that give rise to this disorder have been reported ${ }^{4,5}$ : a heterozygous 277G-A transition (c.277G $>$ A; p.G93S) and a 313C-G transversion (c.313C $>$ G; p.Q105E), both in exon 2. Homozygous mutations in the OPA3 gene have also been reported and are responsible for recessive 3-methylglutaconic aciduria type III or Costeff optic atrophy syndrome (3-MGCA: MIM 2585016 ${ }^{6}$ ). Costeff syndrome is a neuro-ophthalmic syndrome characterized by increased urinary excretion of 3-methylglutaconic acid and 3-methylglutaric acid, with early-onset bilateral optic atrophy, reduced visual acuity, and later-onset spasticity, extrapyramidal dysfunction, and occasional cognitive defect. ${ }^{7}$ Three homozygous mutations have been reported in OPA3. The first mutation to be reported was homozygous in all patients of IraqiJewish origin and is a splice site founder mutation, c.143-1G $>$ C (IVS1-G $>$ C) that abolishes mRNA in fibroblasts. ${ }^{6}$ An in-frame 18-bp deletion in exon 2, c.320_337del (p.Q108_E113del), ${ }^{8}$ and a homozygous nonsense mutation, c.415C $>$ T (p.Q139X), also in exon 2,9 have also been reported.

Databases show that OPA3 consists of a 5' UTR, an open reading frame encoding 179 amino acids, and $>970$ nucleotides of 3 ' UTR sequence spanning approximately $57 \mathrm{~kb}$ on human chromosome 19 , region q13.2-q13. ${ }^{6}$ Recent evidence shows that there are two alternate transcripts, $O P A 3 A$ and $O P A 3 B,{ }^{10}$ arising from alternate splicing of a third exon. OPA3 is a novel protein with no known homolog, and therefore no useful predictions of tertiary structure can be made from bioinformatics databases. It is predicted to be a $20-\mathrm{kDa}$ peptide containing a mitochondrial leader and targeting sequence (NRIKE) at amino acid residues 25-29 of the $\mathrm{N}$ terminus (http:// psort.nibb.ac.jp/ a collaborative database of Predictions of Protein Sorting Signals and Localization Sites in Amino Acid Sequences) and a putative peroxisomal sorting tripeptide signal at the C-terminal. ${ }^{11}$ Green fluorescent protein-tagged OPA3 expression studies have found it to be localized predominantly to mitochondria. ${ }^{10}$ Proteomic studies have shown that the $O P A 3$ gene encodes a mitochondria-related protein that may localize to the inner mitochondrial membrane. ${ }^{12}$ However, subcellular fractionation of cultured cell lines suggests rather that OPA3 localizes to the outer mitochondrial membrane 
A

B

Gene

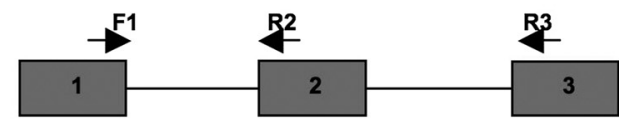

cDNA

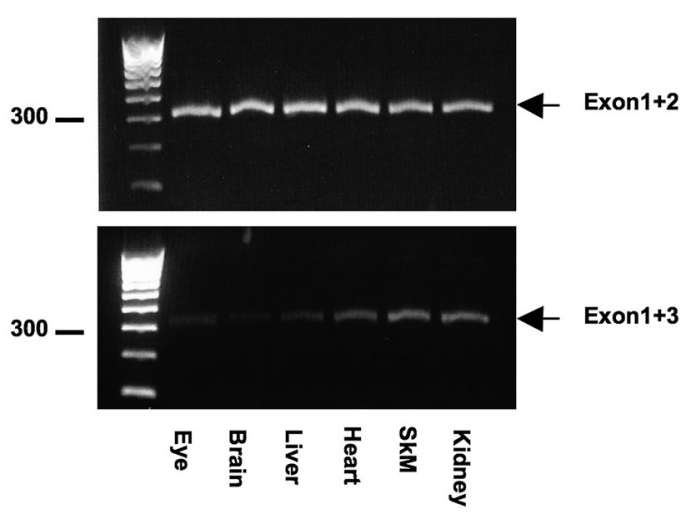

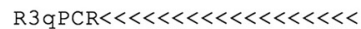

$>>>>>>>>>>>>>>P E 1$

Exon1-2 ATGGTGGTGGGCGCGTTCCCCATGGCGAAGCTGTTCTACTTGGGCATCCGGCAGGTTAGC 60 Exon1-3 ATGGTGGTGGGCGCGTTCCCCATGGCGAAGCTGTTCTACTTGGGCATCCGGCAGGTTAGC 60

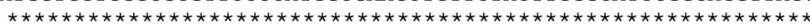

Exon1-2 AAGCCGCTGGCCAACCGTATCAAGGATGCTGCCCGCCGCAGCGAGTTCTTCAAGACCTAC 120 Exon1-3 AAGCCGCTGGCCAACCGTATCAAGGATGCTGCCCGCCGCAGCGAGTTCTTCAAGACCTAC 120

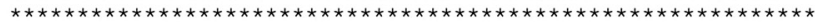

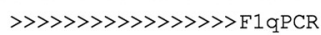

Exon1-2 ATCTGCCTACCGCCAGCCCAGCTGTACCACTGGGTGGAGATGCGGACGAAGATGCGCATA 180 Exon1-3 ATCTGCCTACCGCCAGCCCAGCTGTACCACTGGCTAGAGATGCGGACCAAGATGCGCATC 180

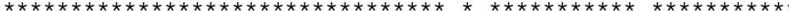

Exon1-2 ATGGGTTTCCGGGGCACTACCATCAAGCCACTGAATGAGGAGGCAGCAGCAGAGCTGGGT 240 Exon1-3 ATGGGCTTCCATGCTGAGGCCATCAAGCCACTGAATGAGGATGCAGCGGCTGAGCTGGGT 240

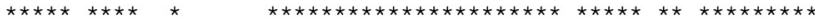

Exon1-2 GCTGAGCTGCTGGGCGAGGCCACCATCTTCATTGTGGGCGGAGGCTGCCTGGTCCTGGAG 300 Exon1-3 GCGAACCTGCTGGGTGAAGCCATCATCTTTGCTGCTGCGGGCAGCTGTCTGCTGCTGGAG 300

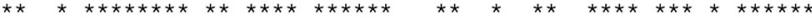

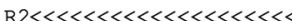

Exon1-2 TACTGGCGCCACCAGACTCAGCAGCGCAATAAGGAGGAGGAACAGCGGGCAGCCTGGAAC 360 Exon1-3 TTCTGGCGCCAGAAGTCATCGAAACACCGCAGGGAAGTGGCGCAGGTGGC--CACGGTGC 358

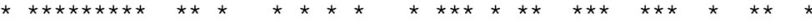

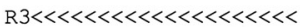

Exon1-2 GCTC--TGCAGGATGAGGTGGGCCGCCTGGCACTGGCCTTAGAGGCGCTGCAGGCTCAGG 418 Exon1-3 TGTCGCTGCGGGAGGATGTGGAATACCTGGAGAACATGCTGGACGAGGTGCAGGTGCAGG 418

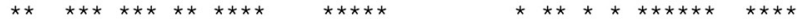

Exon1-2 CACAAGCGA---TGCCC------TCCTTGAGTGCCCTGGAGG---AGCTGCGGGAGGAGC 466 Exon1-3 TGCAGGCAGCATTGCCCAGGAACTCACTGGATGAGCTGCGGGCAGAGCTGCGGGCAGAGC 478

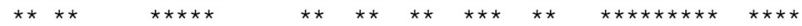

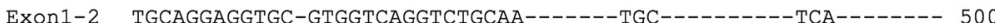
Exon1-3 TGCGGGCGGAGCTGCGGACAGAGTTGCAAGCAGAGTTGCGGGCAGAGCTTCAAGATGAGC 538

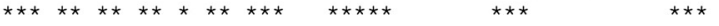

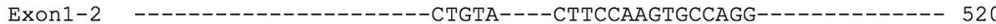
Exon1-3 TACAGAAGTTCAGAACCCAGATCTGTAAAGATTGCTATGAGCCAGAGCTTAAGCCTGAAC 598

Exon1-2 --CAGCATCTT-------CCAAGAAATAG 540

Exon1-3 TCCAGTGTCCTGAGGCCCCCAAGGAGTAG 627

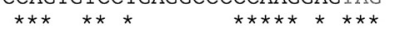

C Opa3 transcripts a and b Vs Opa 3 transcript b expression in Lens

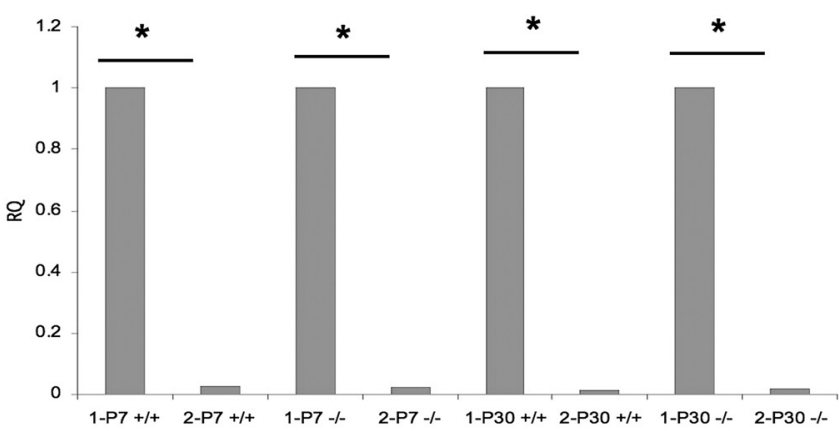

D Opa3 transcripts a and b Vs Opa3 transcript b expression in Retina

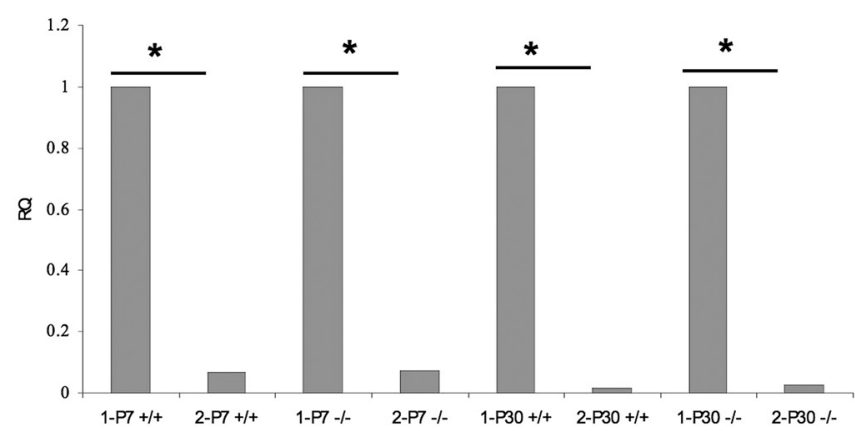

FIGURE 1. 
(MOM), ${ }^{13}$ with a $\mathrm{C}$ terminus exposed to the cytosol and an $\mathrm{N}$-terminal mitochondrial targeting domain.

Fibroblasts obtained from patients with ADOAC have been shown to be particularly susceptible to apoptotic stimuli, although, no abnormalities in the respiratory chain, mitochondrial membrane potential, or morphology of the mitochondrial network were identified in these fibroblasts. ${ }^{4}$ However, Chevrollier et al., ${ }^{14}$ in studies of fibroblast cell lines, found reduced membrane potential and a possible coupling defect in OXPHOS. Overexpression of the G93S human OPA3 mutation in HeLa cells increased cell death and mitochondrial fragmentation in the absence of apoptotic stimuli. ${ }^{13}$ A model of 3-methylglutaconicaciduria type III (3-MGA) recently reported in Opa3 null allele zebrafish, ${ }^{15}$ demonstrates normal mitochondrial oxidative phosphorylation, but increased sensitivity to inhibitors of the electron transport chain.

We have reported a mutant mouse $\left(\mathrm{B} 6 ; \mathrm{C} 3-\mathrm{Opa} 3^{\mathrm{L} 122 \mathrm{P}}\right)$ that carries a missense point mutation in exon 2 (c.365T $>C$; p.L122P). ${ }^{16}$ Homozygous mice display a condition with many similarities to the recessive 3-MGA type III disorder observed in patients: impaired visual function consistent with loss of retinal ganglion cells and degeneration of the optic nerve, elevated serum organic acids, dilated cardiomyopathy, extrapyramidal dysfunction, and gross neuromuscular defects. Here, we report the expression of murine Opa3 splice variant transcripts and Opa3 protein in development and maturation in a range of tissues-most significantly, lens and retina in homozygous mice carrying the p.L122P mutation. We studied mouse embryonic fibroblasts deriving from these animals and localized Opa3 to the mitochondria. We used electron microscopy to assess mitochondrial morphology in retinal tissues from Opa3 mutant mice.

\section{Materials AND Methods}

\section{Splice Variant Analysis}

For RT-PCR to detect alternately spliced Opa3 isoforms in tissues, total RNA was isolated from mouse eye, brain, liver, heart, skeletal muscle, and kidney of adult wild-type mice (RNeasy Mini kit; Qiagen, Crawley, UK). cDNA was synthesized (Quantitect Reverse Transcription Kit; Qiagen), and RT-PCR was performed with primers in the Opa3 exon coding regions spanning introns, close to exon-intron boundaries. The primer sequences were F1: 5'-GGCGAAGCTGTTCTACTTGG-3' (exon 1); R2: 5'-TTATTGCGCTGCTGAGTCTG-3' (exon 2); and R3: 5'-GCGGTGTTTCGATGACTTCT' (exon 3) (Fig. 1A). (No-reverse-transcription enzyme, genomic DNA, and water-only reactions were performed as controls.) Transcripts 1-2 and transcripts 1-3 were sequenced (primers available on request). PCR products were purified for sequencing (QuickClean; BioLine, San Francisco, CA). All PCR products were sequenced in forward and reverse to confirm the sequence. Mouse exon alignments were performed by Clustal W. ${ }^{17}$

\section{Quantitative RT-PCR}

Three different samples of both lens and retina at both $\mathrm{P} 7$ and $\mathrm{P} 30$ from three wild-type and three homozygous mutant Opa3 mice were harvested and placed in RNA stabilizer (RNAlater; Ambion, Austin, TX).
Total RNA was isolated (Trizol Reagent; Invitrogen, Carlsbad, CA) and purified (RNeasy Clean Up kit; Qiagen). One microgram of total RNA from each sample was reverse transcribed (High Capacity cDNA reverse transcription kit; Applied Biosystems, Inc. [ABI], Foster City, CA). Quantitative PCR reactions were prepared (TaqMan Universal PCR Master Mix, No AmpErase UNG; ABI) and mixed with cDNA, primers and the probe gene-specific assay mix. Gene expression assays (TaqMan; ABI) were used for Mus musculus Opa3 (assay ID: Mm01702385_ml; (90-bp amplicon) and Mus musculus Sod2 superoxide dismutase 2 (assay ID: Mm00449726_ml; 67-bp amplicon; both from $\mathrm{ABI}$ ). The primer sequences for $\mathrm{Opa} 3$ are made on the exonexon junction (Assay ID: Mm01702385_ml) and detect both transcripts. Primers for exon 1_3 transcript were designed in-house as F1 qPCR: 5'-CCAGCCCAGCTGTACCA-3 and R3 qPCR: 5'-TTGATGGCCTCAGCATGGAA-3 (which is specific for transcript b; Fig. 1A). qPCR was performed in triplicate ( $7900 \mathrm{HT}$ Prism; ABI), with the mean CT values used to calculate the relative gene expression levels after normalizing to $18 \mathrm{~S}$ RNA levels (endogenous control: VIC/MGB probe; Primer Limited; ABI). Analyses of relative gene expression data were performed using the $2{ }^{\Delta \Delta} C_{\mathrm{T}}$ method. ${ }^{18}$ Statistical analysis was performed with the Tukey post hoc test and expressed as ANOVA $P$ values.

\section{Tissue and Developmental Expression}

Total RNA was isolated from whole wild-type mouse embryos at embryonic day (E)8 and E10 and from body or head homogenates at E13, E15, E17 and postnatal day (P)0 (RNeasy Mini kit; Qiagen). Retina, lens, and optic nerve were dissected from adult wild-type eyes (P30). Firststrand cDNA synthesis was performed with kit (BioLine) with Oligo $(\mathrm{dT})_{18}$ primers. PCR was performed using mOpa3 primers that do not distinguish between transcript $a$ and transcript $b$, designed to span an intron-exon boundary (forward 5'-GGCGAAGCTGTTCTACTTGG-3; reverse 5 '-CTGCTGCCTCCTCATTCAGT-3) giving a final product of 202 bp.

\section{Isolation and Culture of Mouse Embryonic Fibroblasts and Cell Lines}

Embryos from Opa3, with or without crosses of G4 mice at E12.5, were used to make wild-type and $\mathrm{Opa} 3^{-/-}$mouse embryonic fibroblast cell lines. (All experimental work with animals used in this research adhered to the ARVO Statement for the Use of Animals in Ophthalmic and Vision Research). Genotyping for Opa3 was performed according to published protocols. ${ }^{16}$ The fibroblastic cell mass was dissociated with a combination of trypsin and mechanical disruption. Cell slurries were cultured in Dulbecco's complete medium with high glucose (Invitrogen) supplemented with $10 \%$ heat-treated fetal calf serum, $2 \mathrm{mM}$ glutamine, and $100 \mu \mathrm{g} / \mathrm{mL}$ primocin (Invitrogen). Vero, RGC-5, and Brl cell lines were cultured in Dulbecco's complete medium (Invitrogen) supplemented with $10 \%$ fetal calf serum, $2 \mathrm{mM}$ glutamine, $100 \mathrm{U} / \mathrm{mL}$ penicillin, and $100 \mu \mathrm{g} / \mathrm{mL}$ streptomycin.

\section{Antibodies}

Three different commercially available antibodies to OPA3 were used in this study. A mouse polyclonal raised against full-length human OPA3 (H00080207-B01P; Abnova-Novus Biologicals, Cambridge, UK), a rabbit polyclonal raised against human OPA3 24-179 (15,638-1-AP ; ProteinTech Group, Chicago, IL), and a goat polyclonal raised against

Figure 1. Opa3 has two splice variants in mice, which are ubiquitously expressed. (A) Comparison of murine Opa3a (exon1-2) and Opa3b (exon1-3) transcripts. Transcripts show an $81 \%$ sequence identity. Blue: exon 1; black: alternative second exons; red: first stop codon in each transcript. Alignment was generated by the ClustalW2 program. (*) The residues or nucleotides in that column are identical in all sequences in the alignment. F1, R2, and R3 show position of primers for identification of splice variants. (B) RT-PCR showing tissue expression of alternately splice variants of murine $O p a 3$ in a panel of tissues (eye, brain, liver, heart, skeletal muscle, and kidney). Gene: the Opa3 gene, showing the location of RT-PCR primers used to verify third exon and splice variant. cDNA: exon 1 splices to exon 2, giving a product of $310 \mathrm{bp}(1+2)$, or to exon 3 giving a product of $307 \mathrm{bp}(1+3)$. Primers F1 and R3 did not give rise to a transcript product of Exons $1+2+3$, suggesting that they are not spliced. (C, D) qPCR showing relative expression of Opa3 transcripts a and b versus transcript a in lenses and retina from wild-type $(+/+)$ or homozygous mutant $(-/-)$ animals at $\mathrm{P} 7$ and $\mathrm{P} 30 .{ }^{*} P<0.001$. 
an internal epitope of human OPA3 (E15, sc-82004; Santa Cruz Biotechnology, Santa Cruz, CA).

Other antibodies used were: PMP70, raised in rabbit (an3421; Abcam, Cambridge UK), COX IV (HRP conjugate), mouse monoclonal (ab62164; Abcam), $\beta$-actin mouse monoclonal, clone AC-15 (AV5441; Sigma-Aldrich, Poole, UK), MnSOD raised in rabbit (ab13533; Abcam), and Porin rabbit polyclonal (Ab-5; Merck, Darmstadt, Germany).

\section{Mitochondrial Visualization, Immunofluorescence Staining, and Microscopy}

Mitochondria were visualized by staining in live cells with the fixable fluorescent red probe (MitoTracker Red CMXRos; Invitrogen, Paisley, UK). The cells were seeded at the appropriate density onto poly-Dlysine-coated glass coverslips and allowed to recover for 24 hours before visualization by incubation with the red probe at a final concentration of $100 \mathrm{nM}$ added to the culture medium for approximately 15 minutes. Cells, with or with the probe, were fixed and permeabilized in cold methanol $\left(-20^{\circ} \mathrm{C}\right)$ for 10 minutes. Washes were performed with PBS containing $0.05 \%$ saponin. Nonspecific sites were blocked with 5\% normal goat serum in PBS containing saponin (PBS/S) for 1 hour at room temperature. Incubation with primary antibody diluted in $\mathrm{PBS} / \mathrm{S}$ containing $1 \%$ normal goat serum was performed overnight at $4{ }^{\circ} \mathrm{C}$ and visualized using fluorescent secondary antibodies (AlexaFlour 488 or $594 \mathrm{~F}^{\left(\mathrm{ab}^{\prime}\right)^{2}}$ fragments of IgG $(\mathrm{H}+\mathrm{L}$; Invitrogen) diluted in PBS/S for 1 hour at room temperature. In some preparations, nuclei were counterstained with aqueous Hoechst $33342(1 \mu \mathrm{g} / \mathrm{mL})$. Coverslips were mounted using an aqueous antifade mounting medium (Fluosave; Calbiochem, San Diego, CA). Immunostaining of Opa3 was performed using either the MaxPab mouse polyclonal antibody (Abnova) or rabbit polyclonal antibody (ProteinTech Group). Peroxisomes were labeled with an antibody to PMP-70. The images were acquired with a fluorescence microscope (DM6000; Leica, Wetzlar, Germany) equipped with a $63 \times / 1.4$ oil differential interference contrast [DIC] objective (Plan Apochromat; Carl Zeiss Meditec, Oberkochen, Germany).

\section{Western Blot Analysis}

Cell or tissue lysates were prepared by homogenization in RIPA buffer containing a protease inhibitor cocktail (Sigma-Aldrich) on ice, followed by brief centrifugation to remove debris. Reducing SDS-PAGE was run routinely with Laemmli sample buffer containing a final concentration of $100 \mathrm{mM}$ DTT up to a maximum concentration of $400 \mathrm{mM}$. Subcellular fractionation was performed on cultures of mouse embryonic fibroblasts derived from wild-type or homozygous embryos. A total of $2 \times 10^{7}$ cells per sample were lysed and separated into fractions with a mitochondrial isolation kit (Qproteome; Qiagen). Samples were collected from each stage of the process (cytosol, microsome, and mitochondrial) and desalted and concentrated by acetone precipitation, and protein concentration was determined. Equal amounts of protein (50 $\mu \mathrm{g}$ per lane) were loaded for each sample. SDS-polyacrylamide electrophoresis was performed on $12 \%$ polyacrylamide gels (37.5 acrylamide: 1 bis-acrylamide; Applichem; Merck) and the separated proteins transferred to nitrocellulose membrane $(0.2 \mu \mathrm{m}$ pore size; Bio-Rad, Hemel Hempstead, UK). A variety of primary antibodies were used to probe blots: OPA3 MaxPab mouse polyclonal antibody (Abnova), OPA3 rabbit polyclonal (ProteinTech Group), OPA3 goat polyclonal (not shown), MnSOD rabbit polyclonal, Porin/ VDAC rabbit polyclonal, COX IV horseradish peroxidase (HRP)-conjugated mouse polyclonal, and PMP-70 rabbit polyclonal. Detection was by ECL using HRP-conjugated goat anti-mouse or goat anti-rabbit IgG $(\mathrm{H}+\mathrm{L})$ secondary antibodies (AffiniPure $\mathrm{F}\left(\mathrm{ab}^{\prime}\right)_{2}$ Fragment; Jackson ImmunoResearch Europe, Ltd., Newmarket, UK), and ECL substrate (SuperSignal West Pico; Thermo Scientific-Pierce, Rockford, IL) exposed to clear blue x-ray film (CL-Xposure; Thermo Scientific).

\section{Histology and DAB Immunohistochemistry}

Eyes were removed whole, and the capsule was carefully scored with a razor blade to enable penetration of the reagents. The tissue was fixed in fresh 4\% PFA in PBS, dehydrated through a prolonged series of alcohols, and embedded in paraffin wax. Sections were mounted on coated slides (Histobond; R.A. Lamb, Ltd., East Sussex, UK). Antigen retrieval was performed by heating the slides in $10 \mathrm{mM}$ sodium citrate buffer ( $\mathrm{pH}$ 6.0) for 10 minutes and/or digestion with $0.1 \% \alpha$-chymotrypsin (Sigma-Aldrich). Nonspecific binding sites were blocked with $5 \%$ normal goat serum, $1 \%$ fatty acid-free fraction V BSA, $0.1 \%$ fish skin gelatin, and $0.1 \%$ triton X-100 in PBS for 1 to 2 hours at room temperature. Anti-OPA3 primary antibody (ProteinTech Group) was diluted in PBS containing $1 \%$ normal goat serum and incubated overnight at $4{ }^{\circ} \mathrm{C}$. Washes were performed with PBS containing 0.05\% Tween 20 (PBS/T). Sections were incubated with biotinylated secondary antibody (Stratech, Newmarket, UK) diluted in PBS/T for 1 hour at room temperature, followed by antifade reagent (RTU Vectastain ABC reagent, PK-7100; Vector Laboratories, Peterborough, UK) for 30 minutes at room temperature and then DAB substrate (DAB substrate kit for peroxidase; Vector Laboratories). The sections were counterstained with Harris hematoxylin (Thermo Scientific) and the images acquired (DMRA2 microscope with QWin software; Leica).

\section{Electron Microscopy}

$\mathrm{Opa}^{-/-}$mice and wild-type littermate controls were perfusion fixed with a mixture of $4 \%$ paraformaldehyde and $5 \%$ glutaraldehyde in 0.1 M sodium cacodylate buffer at weaning (age 22 days). The eyes were dissected, and small pieces of retina were removed and postfixed with $1 \%$ osmium tetroxide. The sections were poststained with uranyl acetate and lead citrate, and representative micrographs were taken across the retina at $5000 \times$ magnification.

\section{Bioinformatics}

To identify evolutionarily conserved regions (ECRs), the $5^{\prime}$ untranslated region of $O p a 3$ was compared in a range of species by using Mulan software ${ }^{19}$ (http://mulan.dcode.org/ sequence-alignment software developed by a collaborative group). A region of $>100 \mathrm{bp}$ and $>70 \%$ identity cutoff was specified, which is the conventionally accepted stringency for identification of functional mammalian regulatory elements. ${ }^{20}$ The -5000 - to +280 -bp genomic sequence of mouse Opa3 was compared with that of corresponding human, rat, dog, cow, Macaca, and chimp sequences (5 $\mathrm{kb}$ is the approximate distance between $O p a 3$ and the nearest upstream gene, Grp4, in the mouse). Mulan's graphic alignment used the mouse sequence as the reference sequence. The results were submitted to MultiTF (http://multitf.dcode. org/ a database allied with pSORT) using the "optimized for function" search option of MultiTF to identify conserved transcription-factor consensus sequences. A large number of possible transcriptional factor-binding sites (TFBSs) were identified. We focused analysis only on the sequences that are evolutionary conserved upstream of Opa3. The sequence data from the conserved regions identified by alignment with Mulan was entered into commercial software ${ }^{21}$ (Matinspector; Genomatix, Munich, Germany) ${ }^{21}$ and TFsearch (http://www. cbrc.jp/research/db/TFSEARCH.html) with a core similarity cutoff value set at $\geq 0.9$. We also used PreMod Mouse Mar. 2005 assembly (mm6, Build 34; http://genomequebec.mcgill.ca/premod/ McGill University, Montreal, Quebec, Canada) for genome-wide cis-regulatory module (CRM) predictions. Our selection of programs and high stringencies were chosen to complement each other for a more robust and accurate prediction of TFBSs in the $5^{\prime}$ region.

\section{Results}

\section{Murine Opaßa Is Highly Expressed in Lens and Retina in $\mathrm{Opa}^{-/-}$Mice}

Murine $O p a 3$ consists of three exons. Exon 1 is 181 bp (Ensembl position Chr7 19,813,753-19,813,933; Ensembl genome browser, The Sanger Center, Cambridge, $\mathrm{UK}^{22}$ ) and exon 2 is 398 bp (Ensembl position 19,830,103-19,830,501), with an 
exon 1 to 2 intronic sequence of 16,169 bp. Opa3 exon 3 is 469 bp in mouse (Ensembl position Chr 7 19,840,705$19,841,173$ ) with an exon $2-3$ intronic sequence of $26,771 \mathrm{bp}$. Comparison of the mouse exon 3 sequence with mouse exon 2 sequence showed a $61 \%$ sequence identity. The murine transcript a and transcript $b$ sequence that we generated is given in Figure 1A. Primer pairs spanning exons 1-2 and exons 1-3 yielded products compatible with two predicted splice variants, transcripts $a$ and $b$. Transcript a (exon 1-2) and transcript b (exon 1-3) were expressed in all six wild-type mouse tissues tested. Exons 2 and 3 do not splice (Fig. 1B). Murine opa3 therefore has two transcripts, both ubiquitously expressed, similar to findings recently reported in human tissue. ${ }^{10}$ Quantitative PCR assessment of the relative expression of all isoforms versus isoform $b$ in wild-type and homozygous mutant Opa3 $3^{-1-}$ mice at $\mathrm{P} 7$ and $\mathrm{P} 30$ shows that transcript $\mathrm{b}$ is expressed at less than 10-fold levels in lens and retina and that the presence of the mutation does not disturb this ratio (Fig. 1C). The two transcripts are predicted to give rise to two similar peptides, with $61 \%$ sequence identity (shown aligned in Supplementary Fig. S1, http://www.iovs.org/lookup/suppl/ doi:10.1167/iovs.10- 6406/-/DCSupplemental).

\section{Opa3 Is Widely Expressed in Embryonic Development and Adult Ocular Tissues}

Opa3 mRNA was found to be ubiquitous at all time points studied in mouse embryonic development, from E8 to P0. opa3 was present in the lens, retina, and optic nerve of the adult mouse eye (P30; Fig. 2A). The level of protein expressed in eight different tissues from wild-type animals was investigated by Western blot analysis (Figs. 2B, 2C). Whole-tissue lysates from adult animals (equal protein loading by BCA assay; Thermo Scientific-Pierce) were probed for the presence of Opa3 with two different antibodies (mouse and rabbit polyclonal antibodies). Porin was used as a loading control, showing the relative amount of mitochondria in the tissue lysates, rather than the amount of tissue loaded. Opa3 was present in all tissue tested. Under our normal running conditions (Laemmli sample buffer containing $100 \mathrm{mM}$ DTT) in lens, Opa3 predominantly stained a band of $20 \mathrm{kDa}$, as expected, with a much fainter band at approximately $40 \mathrm{kDa}$. In retina, the OPA3 antibody immunostained both bands again, but was more predominant at $40 \mathrm{kDa}$ than at $20 \mathrm{kDa}$. In all other tissues, only the $40-\mathrm{kDa}$ band was detected. This band was most abundant in retina, brain, and skeletal muscle; moderately abundant in liver, heart, and kidney; and just detectable in lung, mirroring the abundance of mitochondria in these tissue lysates as shown by the presence of VDAC/Porin, a mitochondrial outer membrane channel forming protein (Fig. 2B). With high levels of reducing agent (Laemmli sample buffer containing $400 \mathrm{mM}$ DTT), 20-kDa bands were apparent in addition to the $40-\mathrm{kDa}$ band for all mouse tissues tested (Fig. 2C), for a liver mitochondrial fraction, and for a lysate made from cultured HEK (human embryonic kidney) 293 cells.

Immunostaining of wax sections using DAB histochemistry showed that, in the developing eye (E16.5), Opa3 was abundant in the extraocular muscles, anterior epithelial cells of the lens, the cortical lens fibers, and the retina (Fig. 3). Ganglion cell axons were particularly densely stained as they grew into the optic stalk, forming the optic nerve (Fig. 3 inset). In the adult eye, most layers of the retina demonstrated abundant Opa3 immunostaining, with the greatest concentration in the ganglion cells and nerve fiber layer, the inner nuclear layer, and the layer containing the cell bodies of the rods and cones. Opa3 immunoreactivity was also moderately abundant in both the inner and outer plexiform layers and in the inner segments
A

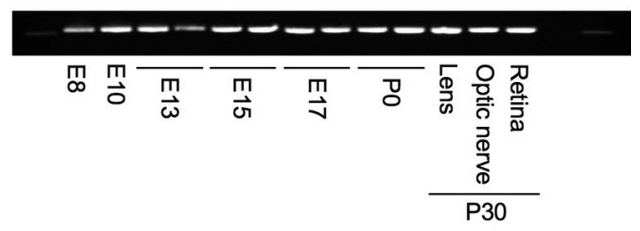

B

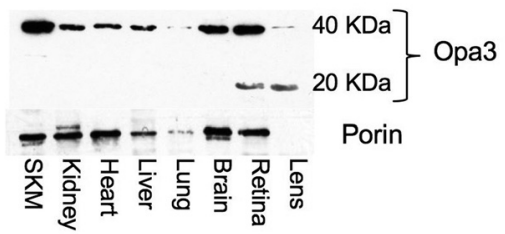

C

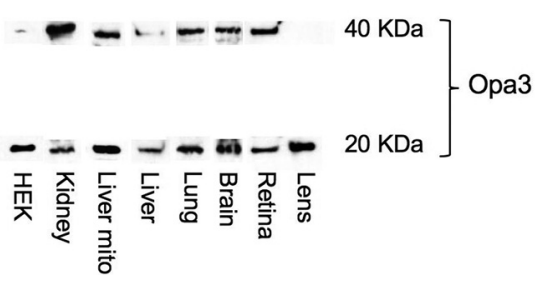

Figure 2. Opa3 mRNA is expressed from early development, and the protein is present in all adult tissues tested. (A) RT-PCR showing expression of Opa3 during embryonic development in whole embryos from E8 to E10, in head and body subsets of embryos from E13 to birth, and in adult (P30) lens, retina, and optic nerve. (B) Western blot of Opa3 protein expression in a panel of mouse tissue lysates (skeletal muscle, kidney, heart, liver, lung, brain, retina and lens). In the blot shown, rabbit anti-Opa3 antibody was used and detects bands at 20 and/or $40 \mathrm{kDa}(t o p)$. Bottom: VDAC/Porin as a mitochondrial marker. (C) Western blot of Opa3 protein expressed in human embryonic kidney cells (HEK) and in a panel of mouse tissue lysates (kidney, liver mitochondrial fraction, liver, lung, brain, retina, and lens), using increased reducing agent in sample buffer. Rabbit anti-Opa3 antibody detected bands at 20 and $40 \mathrm{kDa}(t o p)$. (Composite image from more than one gel.)

of the photoreceptor layer (Fig. 4A). Abundant staining was seen in the extraocular muscles (Fig. 4B). In the cornea, both epithelial and endothelial cell layers were Opa3 positive (Fig. 4C); however, Opa3 immunoreactivity was absent from the corneal stroma. In both immature (Fig. 4D) and adult (Fig. 4E) lenses, Opa3 staining was most abundant in the cuboidal lens epithelial cells, which cover the anterior portion of the lens, and in the lens fiber cells lying underneath this epithelium (Fig. 3). Staining was also relatively abundant in the cortical region of the lens, although it was absent from a band of cortical secondary fiber cells and was visible in the region where the organelle-free zone forms (i.e., where mitochondria are actively being degraded). It was absent from the nuclear fibers and the organelle-free zone, which develop at the center of the lens as it matures.

\section{Validation of OPA3 Antibodies}

The presence of a higher molecular size band $(\sim 40 \mathrm{kDa})$ in immunoblots of mouse tissue was unanticipated. In all three different commercially available antibodies were used in an attempt to validate this result. All three antibodies detected 20-kDa bands in immunoblots of lens and retina tissue, with an additional approximately $40-\mathrm{kDa}$ band also present in lens and retina (Fig 2). In all other tissues tested, only the $40-\mathrm{kDa}$ band was detectable. Staining of both bands in immunoblots (and of 


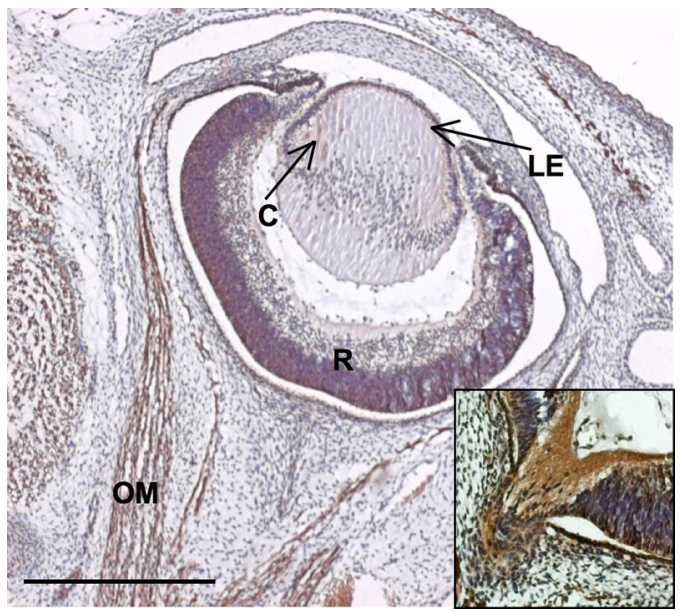

Figure 3. Opa3 is present in the developing eye. DAB immunohistochemistry of wax sections from E16.5 mouse embryos (main figure). Sections immunostained with a rabbit polyclonal antibody to Opa3 labeled with DAB (brown) and counterstained with hematoxylin (blue). Inset: the developing optic nerve from E14.5 embryo showing migration of retinal ganglion cell axons into the optic nerve. LE, lens epithelium; LC, cortical lens fibers; R, retina; OM, extraocular muscle Scale bar, $50 \mu \mathrm{m}$.

sections or cells in immunocytochemical or immunohistochemical procedures) was readily abolished by preabsorption of the antibody with appropriate peptides. In immunofluorescence experiments, Opa3 immunostaining was localized to the mitochondrion in several of the species (Fig. 5), and by Western blot, Opa3 was also detected in the mitochondrial fraction (Fig. 6). Whole-tissue lysates and mitochondrial-enriched subcellular fractions derived from the corresponding tissue detected bands of the same size $(\sim 40 \mathrm{kDa})$, but with a stronger signal from mitochondrial fractions, as would be expected with a protein thought to localize within the mitochondrion.

\section{Mutant $O \mathrm{pa}^{-/-}$Localizes to Mitochondria and Disrupts Mitochondrial Architecture}

Opa3 immunostaining was localized to the mitochondria in mouse embryonic fibroblasts derived from wild-type (Fig. 5A) or mutant (Fig. 5D) animals. Mitochondrial localization was verified by counterstaining with a fixable vital dye (MitoTracker Red CMXRos; Invitrogen). Figure 5A shows that most of the Opa3 signal co-localized with red-stained mitochondria to produce an orange co-localization signal (the inset shows a higher magnification of the mitochondria). The pattern of mitochondrial localization within the cell (Fig. 5B; CMXRos stain; Invitrogen) can be compared with the pattern of immunostaining obtained with an antibody to OPA3 (Fig. 5C). Localization of Opa3 immunostaining did not affect the presence of the mutation in embryonic fibroblasts derived from homozygous mutant animals (Figs. 5D-F). However, co-localization of Opa3 with the mitochondria is not total in either wild-type or mutant cells, and although all mitochondria contain Opa3 signal, not all Opa3 immunostaining appears to be associated with mitochondrial organelles. This is likely to be due to some measure of nonspecific staining by the antibody, but could indicate a distinct subpopulation of Opa3 within the cell, which is not as yet associated with mitochondrial organelles and may indicate transport vesicles or protein destined for import into the mature mitochondrion. However, the mutant Opa3 protein does not appear to induce any mitochondrial network fragmentation.

Mitochondria are known to serve a variety of functions, which may vary in importance depending on cell type. Some mitochondrial functions are performed only in specific types of cells-for example, mitochondria in liver cells-detoxify waste products of protein metabolism. For this reason, we investigated the distribution of Opa3 in a variety of cell types. Opa 3 immunostaining was present in all cells tested and localized to the mitochondria. These included Vero cells, an epithelial cell line derived from monkey kidney (Fig. 5G), and rat liver Brl cells, a hepatocyte-like, liverderived cell line (Fig. $5 \mathrm{H}$ ). In all cases Opa3 immunostaining co-localized predominantly with the mitochondria. Mitochondria share some roles with the peroxisomes, which are small organelles with diverse, cell-dependent functions that are scattered throughout the cell and play a part in the breakdown of long-chain fatty acids. In addition to these shared roles, other lines of evidence have hinted at the possibility that Opa3 has a functional role in the peroxisome. The Opa3 protein contains a C-terminal tripeptide sequence that has been suggested to share similarity with known peroxisomal targeting sequences. ${ }^{6}$ Some cell lines, including Brl (Fig. 5I) and Mef (Fig. 5J) were immunostained with anti-OPA3 antibody and a peroxisomal-membrane marker (PMP-70). In all cells, immunostaining was separate, and there was no evidence of co-localization of Opa3 with the peroxisome. It therefore appears that Opa3 is an integral protein of the mitochondrion and that any observed nonmitochondrial staining is not derived from an association with peroxisomes.
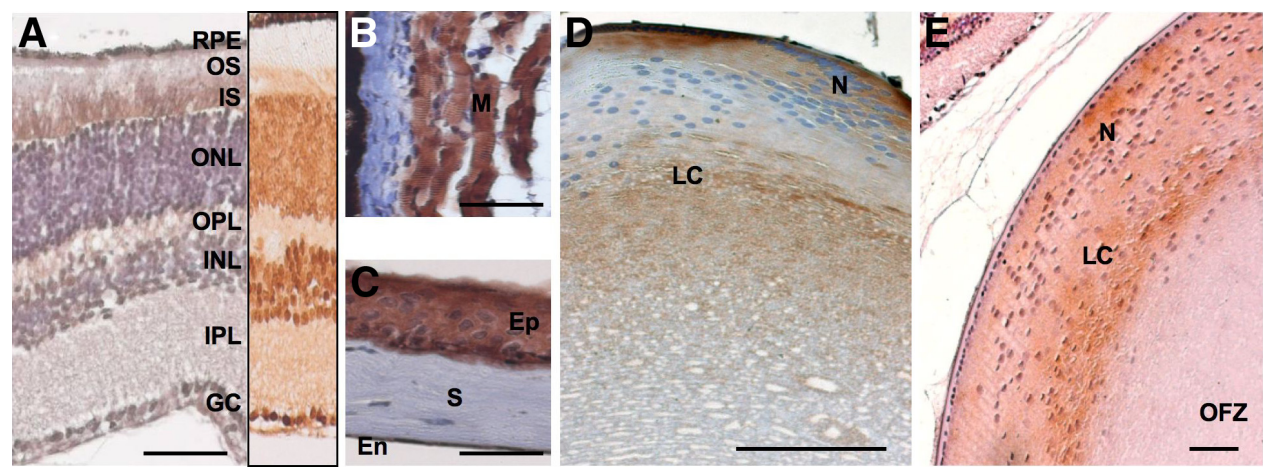

Figure 4. Opa3 was abundant in the ocular structures. DAB immunohistochemistry of wax sections showing the distribution of Opa3 in (A) retina, (B) extraocular muscle, (C) cornea, and (D, E) lens. (A) Section of retina from $\mathrm{P} 7$ mouse, immunostained using an antibody to OPA3 (brown) and counterstained with hematoxylin (blue). Layers of the retina are RPE, retinal pigment epithelium; OS, outer segments; and IS, inner segments of the photoreceptor layer; ONL, outer nuclear layer; OPL, outer plexiform layer; INL, inner nuclear layer; IPL, inner plexiform layer; and GC, ganglion cell. Inset: DAB-stained retina without hematoxylin counterstain. (B) Extraocular muscle (M). (C) Cornea, consisting of epithelial layer (Ep), stroma (S), and endothelial layer (En). (D, E) Lenses from P7 (D) and 9-week-old (E) mice. Blue: nuclei (N). Lens divides into two regions of outer cortical fibers (LC) and inner nucleus (N). As the lens matures, the innermost lens fibers lose all organelles, forming the organelle-free zone (OFZ). Opa3 immunostaining with rabbit polyclonal antibody. Scale bars, $50 \mu \mathrm{m}$. 

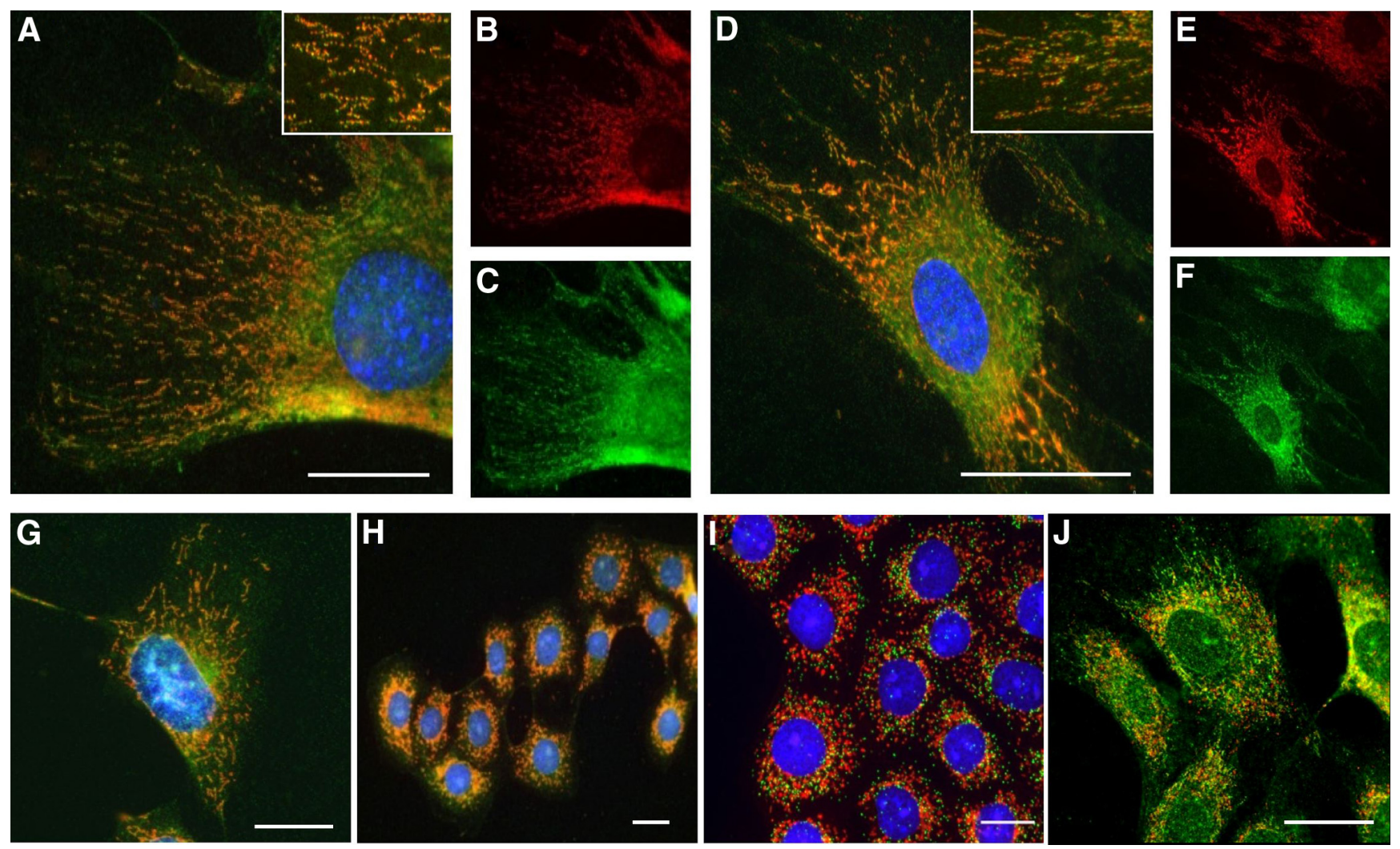

FiguRE 5. Opa3 localized to mitochondria in a variety of cells, but did not colocalize with peroxisomes. Immunostaining with mouse polyclonal anti-human OPA3 antibody. (A) Merged image of an embryonic fibroblast cell derived from a wild-type mouse, immunostained to show Opa3 (green) with mitochondria labeled in vivo using the mitochondrion probe (red). Inset: a higher-power image of dual-stained mitochondria from a different image. (B) Mitochondrial (red) staining or (C) Opa3 (green) immunostaining only. (D) Embryonic fibroblast derived from a homozygous mutant mouse, immunostained as for wild-type (anti-OPA3 green, mitochondrial probe, red). Inset: a higher power image of dual-stained mitochondria from a different image. (E) Mitochondrial staining (red) or (F) Opa3 (green) immunostaining only. (G) Merged image of Vero cell (G) and Brl cell (H) immunostained with an antibody against Opa3 (green) and labeled with the mitochondrial probe (red). (I, J) Merged images of $\mathrm{Brl}$ (I) and wild-type Mef (J) cells immunostained with antibodies against OPA3 and a peroxisomal marker, PMP-70, labeled with a 594 nm-conjugated secondary antibody (red). In all preparations except (J), Hoechst 33342 DNA stain was used to counterstain nuclei (blue). Scale bars: (A, G) $25 \mu \mathrm{m}$; (D-F), $10 \mu \mathrm{m}$.

The mouse model B6;C3-Opa3 ${ }^{\mathrm{L} 122 \mathrm{P}}$, generated by ENU mutagenesis for these studies, has a missense point mutation in the second exon of the gene, giving rise to the substitution of a proline residue for leucine at position 122. A variety of web-based bioinformatics programs were used to predict changes in protein structure that are likely to arise from this substitution and indicated a putative change in the surface accessibility of the protein in this region. Opa3 immunostaining localized to mitochondria in cultured Mef cells derived from wild-type animals. No change in this distribution was apparent in animals homozygous for the mutation in Opa3. Western blot analysis was also used to confirm the mitochondrial localization of Opa3 in subcellular fractions of wild-type and homozygous mutant Mef cells. Equivalent protein concentrations were loaded from four subcellular fractions (nuclear, cytoplasmic, microsomal, and mitochondrial) and were separated by reducing SDS-PAGE. Opa3 immunoreactivity was found only in the band representing the mitochondrial fraction in both wild-type and mutant cells, and the presence of the $\mathrm{L} 122 \rightarrow \mathrm{P}$ mutation did not affect distribution of the protein (Fig. 6A). Opa3 was not detectable in the cytoplasmic or microsomal fractions, suggesting that if Opa3 protein is present in locations other than the mitochondria within the cell, it is at very low levels.

During development, mitochondrial protein expression was not grossly affected by the presence of the Opa3 L122 $\rightarrow \mathrm{P}$ mutation (Fig. 6B). Western blot analysis of lysates from E12.5 embryo heads from wild-type or homozygous mutant animals $\left(\mathrm{Opa} 3^{-1-}\right)$ were probed with antibodies to the mitochondrial membrane protein VDAC/Porin, and to the mitochondrial enzymes Cox VI and MnSOD. Levels of all of these proteins were identical when comparing blots from wild-type and homozygous mutant animals at this time point (densitometry data not shown). Comparison of lysates obtained from embryonic tissue (E12.5) of mice, with or without the Opa3 L122P mutation by Western blot analysis, showed that the relative protein mass of peroxisomes was also unaltered (Fig. 6B). By nonreducing SDS-PAGE, Opa3 was shown to run at a much higher molecular weight, suggesting that it may associate with itself or other proteins to form higher molecular weight complexes in vivo (Fig. 6C). In nonreducing SDS-PAGE, proteins are denatured and coated by SDS to allow passage through the gel, but no reducing agent is added, allowing retention of disulfide bonds that hold subunits and complexes together. The bands containing immunoreactive wild-type and mutant Opa3 run at approximately $200 \mathrm{kDa}$.

Transmission electron microscopy of retinal tissues taken from adult wild-type and homozygous mutant mice revealed subtle alterations to the morphology of mitochondrial cristae in mutants (Fig. 7). Cristae assessed in 21 mitochondria from homozygous animals appeared to be more dilated than in 40 mitochondria from normal, wild-type littermate controls. Sections show comparable mitochondria from the inner nuclear 
A

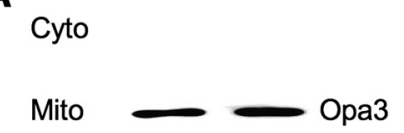

Micro

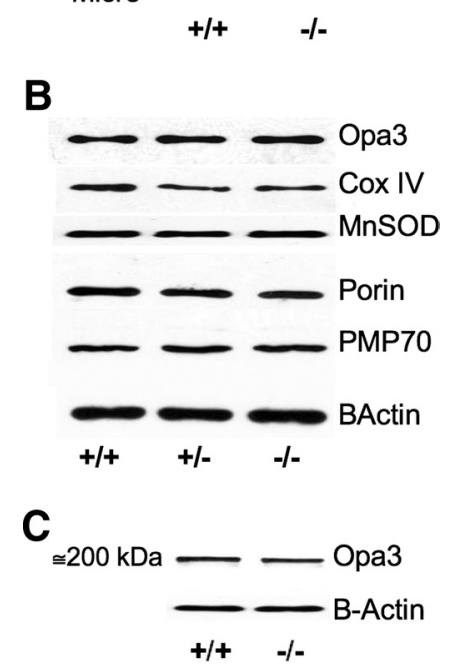

Figure 6. Presence of the Opa3 mutation does not affect its subcellular location, the expression of a panel of mitochondrial proteins or formation of high-molecular-weight complexes. (A) Western blot analysis of subcellular fractions derived from cultured Mef cells. Cells from wild-type or homozygous animals were fractioned with a mitochondrial isolation kit. Equal protein loadings from the cytosolic (cyto) mitochondrial (mito), and microsomal (micro) fractions were separated by SDS-PAGE and immunoblotted, using antibodies against Opa3 (mouse polyclonal in the blot shown). (B) Western blot of lysates from whole embryonic tissue (E12.5) derived from wild-type $(+/+)$, heterozygous $(+/-)$, or homozygous animals $(-/-)$. Blots were probed using a panel of mitochondrial antibodies: Opa3 (rabbit polyclonal in the blot shown), Cox VI, MnSOD, and Porin; a peroxisomal marker, PMP-70, and $\beta$-actin as a loading control. (C) Nonreducing gel of lysates from wild-type or homozygous embryonic tissue (E12.5) immunoblotted using antibodies to OPA3 (rabbit polyclonal in the blot shown), with $\beta$-actin as a loading control.

layer of the retina in wild-type (Figs. 7A-C) or homozygous (Figs. 7D-F) animals.

\section{Opa3 Protein Accumulates in Ageing Lens}

Western blot analysis of lysates from wild-type lenses at birth (P0), $\mathrm{P} 7, \mathrm{P} 14, \mathrm{P} 30$, and $\mathrm{P} 90$ demonstrated that Opa3 protein is present at birth and the protein content increased markedly with age (Fig. 8A). We confirmed that Opa3 was present in the lens at PO, but at much lower levels than in the adult. This increase was mirrored by the mitochondrial outer membrane channel protein VDAC/Porin (Fig. 8A). However, over the same time frame, levels of the mitochondrial matrix antioxidant enzyme MnSOD remained relatively stable (densitometry data not shown) raising the possibility that Opa3, and other membrane proteins such as Porin, accumulate as the lens ages, possibly as a result of slower breakdown and turnover. ${ }^{23,24}$

Comparison of Western blots from age-matched wild-type and homozygous lens lysates (for example, P30; Fig. 8B) showed that the antioxidant enzyme MnSOD was more highly expressed in adult homozygous animals, which was not seen in blots from embryonic tissue. No consistently significant difference was detectable in the levels of Opa3 in wild-type and homozygous lens lysates. However, further investigation of the relative expression of Opa3 mRNA in the lens by real-time quantitative PCR (Fig. 8C) showed that levels of Opa3 cDNA were equivalent at $\mathrm{P} 7$ in both wild-type and homozygous lenses, but that Opa3 cDNA was reduced in wild-type P30 lens $(P=0.012)$ but not in homozygous mutant lenses. There was a statistically significant increase in Opa3 expression in $\mathrm{P} 30$ homozygous mutant mice compared with P30 wild-type ( $P=$ 0.019). The same trend was reflected in the levels of MnSOD in lenses, (Fig. 8D). In week-old (P7) mice, relative levels of MnSOD cDNA, as measured by $\mathrm{qPCR}$, were not significantly different in the wild-type and the homozygous animals. By P30 expression of MnSOD had dropped in the wild-type mice $(P=$ 0.0007 ) but not in the homozygous mutant animals (Fig. 8D). There was a statistically significant increase in MnSOD expression in P30 homozygous mutant mice compared with that in the P30 wild-type $(P=0.004)$. This result suggests that the presence of the Opa3 mutation places an oxidative burden on the older lens, to which cells respond by elevating levels of the mitochondrial antioxidant enzyme.

\section{Putative Transcription Factor Binding Sites Reflect Phenotypic Features of the B6;C3-Opa3 ${ }^{\mathrm{L122P}}$ Mouse}

Multispecies Mulan conservation analysis of exon 1 and the 5-kb region upstream of $O p a 3$ revealed three discrete elements of noncoding evolutionary conserved regions (ECRs) that are conserved in all seven vertebrate lineages using a $>100 \mathrm{bp}$ and $>70 \%$ identity cut off (Fig. 9). These three elements are at -3538 to $-3236 \mathrm{bp},-1326$ to $-1015 \mathrm{bp}$, and -420 to -143 bp. If even more stringent parameters are imposed $(>200 \mathrm{bp}$ and $>77 \%$ identity) only the -1326 to -1015 -bp region is significantly conserved. Mulan alignment between mouse and the six other mammalian species showed nine multiconserved TFBSs in these conserved regions (Fig. 9), all of which except one (RBP-JK) were clustered in the most conserved region of -1326 to $-1015 \mathrm{bp}$. Genomatix Matinspector found six TFBSs in the conserved regions and TFSEARCH identified 18. PreMod produced four CRMs: two upstream and two downstream of Opa3. The two CRMs upstream of Opa3 predicted 29 TFBSs. In total, using several databases, we identified 53 putative TFBSs in the mouse Opa3 5-kb upstream region, and these consequently suggest many putative regulatory proteins that could potentially modulate Opa3 expression (Supplementary Table S1, http://www.iovs.org/lookup/suppl/doi:10.1167/iovs.10-6406/-/ DCSupplemental, and Table 1).

\section{Discussion}

Murine Opa3 gives rise to two splice variants, Opa3a and Opa3b, which are expressed in a wide range of adult mouse tissues and throughout embryonic development. Expression of Opa3b (exon 1-3) was at least a tenth of total Opa3 transcript expression, in both lens and retina. Our findings therefore agree with results obtained in humans. ${ }^{10}$ There are two predicted peptides in mice; however, it has not proved possible to speculate as to the functional significance of these two transcripts, as the Opa3 protein has no known homologies, and therefore bioinformatic tools do not currently provide any meaningful functional predictions. Opa3 was ubiquitously expressed at all time points from E8 to P30 and in all tissues studied, including brain, skeletal muscle, and eye. Of significance to the neuro-ophthalmic features of the disease MGA type III (Costeff) syndrome and to the ADOA and cataract phenotype, Opa3 was expressed in the developing lens and retina and in other ocular structures, such as the extraocular muscles and cornea. Opa3 expression was high in the developing retinal ganglion cells of the optic nerve at E14.5. Introduction of a point mutation into the second exon of Opa3 in 

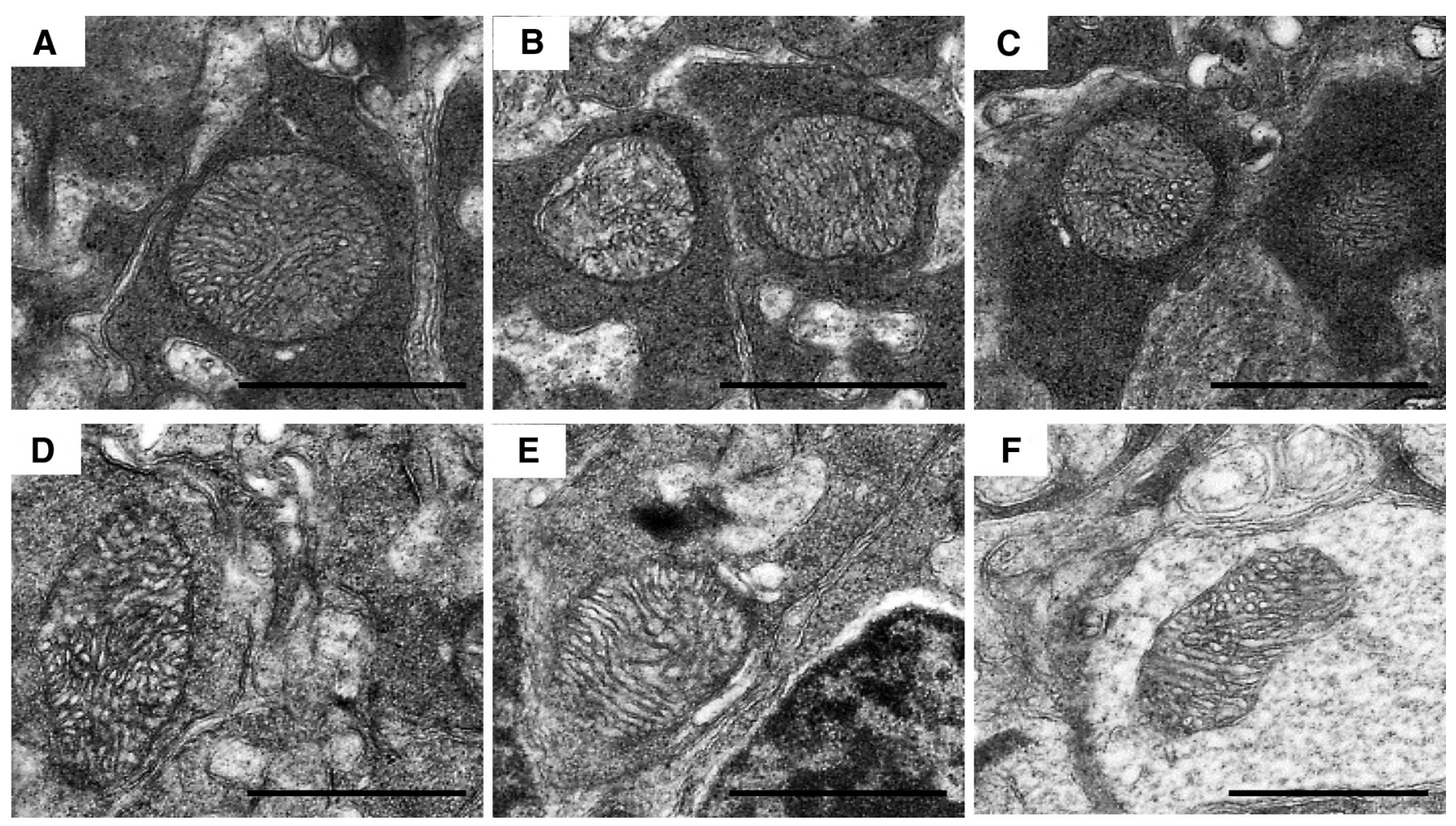

Figure 7. Presence of the Opa3 mutation alters mitochondrial morphology. Representative transmission electron micrographs of mitochondria from retinas of adult wild-type (A-C) and homozygous mutant (D-F) littermate mice, showing that mitochondrial cristae are more disorganized and fragmented in homozygous mutant retina. Scale bar, $1 \mu \mathrm{m}$.

the $\mathrm{B} 6 ; \mathrm{C} 3-\mathrm{Opa} 3^{\mathrm{L} 122 \mathrm{P}}$ mouse gave rise to a phenotype that mimics human recessive MGA type III (Costeff) syndrome. However, the presence of the missense mutation did not alter the relative high expression of Opa3a versus Opa3b transcripts in lens and retina.

To further explore the subcellular localization of Opa3 and the effects of its mutation, we used immunostaining and immunoblot techniques. In this study, three commercially available antibodies to human OPA3 were tested for their ability to recognize mouse opa 3 in several different tissues and using a variety of techniques. These were (1) a mouse polyclonal raised against full-length human OPA3, (2) a rabbit anti-human OPA3 polyclonal antibody raised against a partial peptide sequence from amino acid 24-179, and (3) a goat polyclonal antibody raised against an internal epitope of human OPA3. Two of the antibodies were tested for immunostaining of cells and wax sections: The mouse polyclonal antibody was used for immunofluorescence studies, and gave good results in methanol or acetone-methanol fixed cultured cells of mouse, rat, and monkey origin (Fig. 5). Immunohistochemical staining of wax sections using DAB histochemistry could only be achieved with the rabbit polyclonal antibody (Figs. 3, 4). By Western blot, all three antibodies detected bands at approximately 20 $\mathrm{kDa}$ (the predicted size of the protein) in mouse lens, retina, and all other tissues studied and also at $40 \mathrm{kDa}$ in various intensities in all tissues. The protein product of the Opa3 gene is very similar in human and mouse, particularly in the N-terminal half of the protein, with $84 \%$ to $86 \%$ conserved identity of amino-acid residues in peptide sequences used to raise two of the antibodies tested. Immunostaining (of blots and cells) was abolished when antibodies were preincubated with the appropriate immunizing peptides.

In addition to validation by each manufacturer, the rabbit polyclonal antibody has been independently validated by Owczarek-Lipska et al., ${ }^{25}$ who successfully detected OPA3 in immu- noblots of a human 293T cell line transfected with the bovine protein and in isolated bovine heart mitochondria, although the authors did not report the presence of any higher molecular weight bands. In this study we observed that three different antibodies, raised in different species and to slightly different regions of human OPA3 all give similar results in Western blots of mouse tissue.

The presence of an additional, larger band by Western blot of mouse tissue might arise if the antibody recognizes alternative splice variants. It has been demonstrated that Opa3 has at least two splice variants in human, ${ }^{10}$ bovine,${ }^{25}$ and now in this study, in mouse, which arise by the splicing of exon 1-2 and 1-3, producing transcript $1-2$ and transcript 1-3. However, the splice variants reported to date differ by only a few peptides $(61 \%$ sequence identity; Supplementary Fig. S1, http://www.iovs.org/lookup/ suppl/doi:10.1167/iovs.10-6406/-/DCSupplemental) and would not produce proteins of significantly different sizes. Intriguingly, an early study of Opa $3,{ }^{6}$ in which Northern blot analysis of total RNA was used, demonstrated the presence of two bands, at 5 and $8 \mathrm{~kb}$, which hybridized to the human $O P A 3$ probe and which might therefore suggest the presence of a further transcript. Alternatively, various posttranslational modifications might significantly alter the size of the protein and its running characteristics by SDSPAGE, and this could be differentially regulated. Finally, the protein could form part of a multimeric complex that may not necessarily be completely dissociated by SDS-PAGE. For example, some single-pass transmembrane proteins, such as glycophorin A, can form SDS-resistant dimmers due to the distribution of charge on the protein, such that regions of proteinprotein interaction are internalized and protected from denaturation by SDS. Interestingly, it has been speculated ${ }^{13}$ that Opa3 might be such a single-pass membrane protein. In this study, we shifted Opa3 immunostaining from a single, $40-\mathrm{kDa}$ band seen in most of the mouse tissues, to a combination of $20-$ and $40-\mathrm{kDa}$ bands by the addition of increased 
A

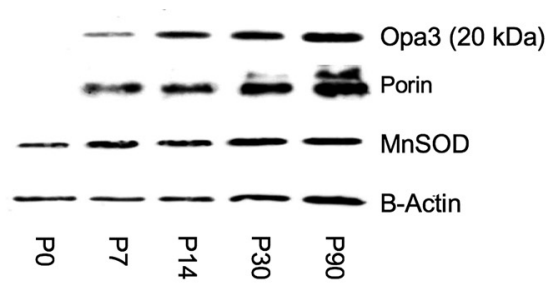

Relative Expression of Opa3 and Porin

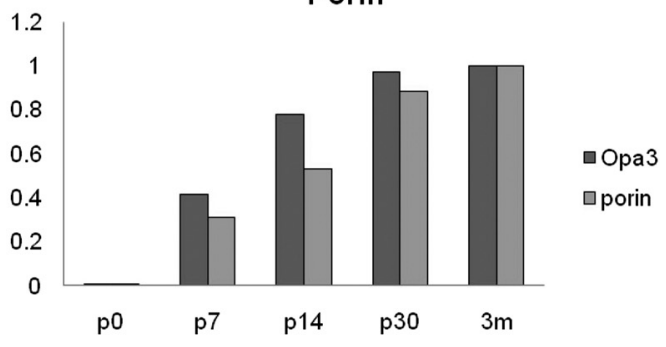

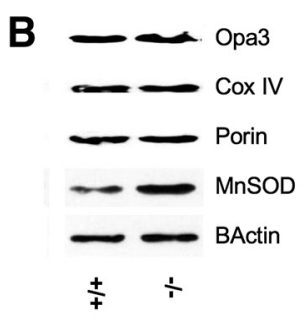

C

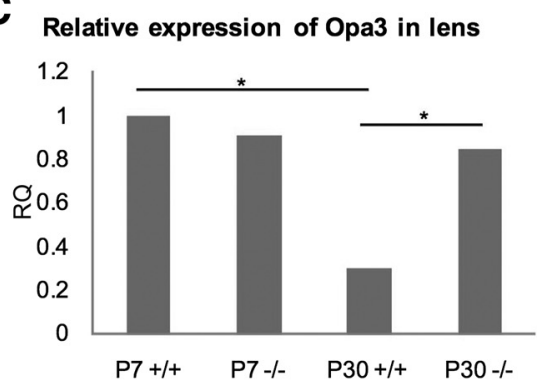

D

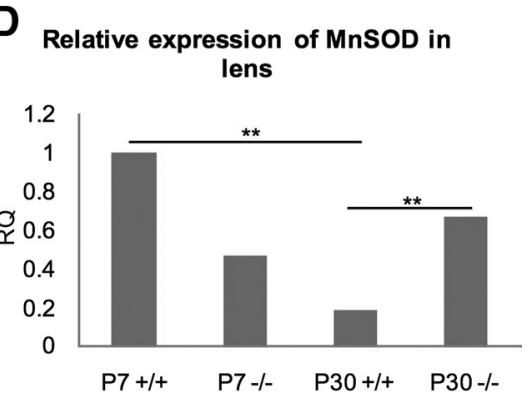

FiguRE 8. Opa 3 protein content increased as the lenses aged. (A) Representative Western blot of lysates from aging wild-type lenses. The blots were probed with antibodies against Opa3 (mouse polyclonal in the blot shown) and antibodies against two known mitochondrial markers, VDAC/Porin and MnSOD, with $\beta$-actin as a loading control. Chart shows densitometry analysis of this immunoblot, normalizing Opa 3 and Porin content to the $\beta$-actin control (B) Cell lysates from wild-type $(+/+)$ or homozygous $(-/-)$ lenses aged 30 days and expression levels of Opa3 protein determined by immunoblotting (mouse polyclonal in the blot shown). Blots were also probed with antibodies against a panel of mitochondrial proteins (Cox IV, VDAC/Porin, MnSOD) and with $\beta$-actin as a loading control. (C, D) qPCR showing relative expression of Opa3 and MnSOD cDNA in lenses from wild-type or homozygous animals at postnatal days 7 and 30. ${ }^{*} P<0.05,{ }^{* *} P<0.005$ amounts of the reducing agent DTT to our sample buffer. We therefore speculate that the $20-$ and $40-\mathrm{kDa}$ bands are both correctly recognized as Opa3 and that the $40-\mathrm{kDa}$ form is a multimeric form of Opa3-possibly a dimer-that requires strongly reducing conditions, to be pried apart.

Wild-type, native Opa3 is localized to mitochondria across a variety of cell types by immunostaining and has also been shown to have mitochondrial localization by subcellular fractionation experiments. We have demonstrated that despite the presence of a putative peroxisomal localization sequence at the $\mathrm{C}$ terminus, ${ }^{6,10} \mathrm{Opa} 3$ does not localize to the peroxisome in any of the cell types tested. Opa3 therefore functions solely within the mitochondrion. The mitochondrial location of Opa3 was not disrupted by the presence of mutant protein in embryonic fibroblast cells derived from the $\mathrm{B} 6 ; \mathrm{C} 3-\mathrm{Opa} 3^{\mathrm{L} 122 \mathrm{P}}$ mouse. No fragmentation of mitochondrial networks was observed in cells from mutant animals, with mitochondria being similar in appearance to those observed in cells derived from wild-type animals. However, electron microscopy of the internal architecture of mitochondria from the retina of mutant mice indicated subtle alterations to cristae morphology.

The presence of the p.L122P mutation does not appear to affect the ability of Opa3 to form multimeric complexes, and Opa3 protein was detected at the same size and in the same quantity in homogenates from embryonic wild-type and mutant tissue. Therefore, the disease traits displayed in animals carrying the Opa3 mutation would not appear to be the result of changes to the protein structure leading to disruption of interactions between Opa3 and itself, or other downstream binding partners.

In this study, we have shown that native Opa3 protein concentration increases during maturation and ageing of the lens. The amplification of levels of Opa3 protein is greatest during the first 2 weeks after birth in mice, but continues to increase thereafter. We have shown that in wild-type animals, mRNA production decreases over the first month in lens, as would be expected, since the lens is forming fibers and the cells are losing their subcellular organelles. Thus, the increased levels of Opa3 must be due to accumulation of the protein, perhaps as cellular processes slow. As development progresses, expression of Opa3 in wild-type and mutant animals appears to be differentially expressed in an age-related manner. By Western blot of lysates from E12.5 embryo heads, levels of Opa3 protein were identical in wild-type and mutant animals, as were the other mitochondrial proteins investigated. Relative expression of Opa3 in the lens by qRT-PCR was also equivalent in wild-type or mutant week-old animals. However, this normal age-related decrease in Opa3 mRNA expression was not seen in 
Figure 9. Mulan alignment revealed evolutionarily conserved regions upstream of Opa3 exon 1. Murine Opa3 sequence was selected as the reference species; the horizontal axis displays the input sequence. Evolutionarily conserved regions were defined as having $>70 \%$ identity, $>100 \mathrm{bp}$. Regions of conserved sequence common to all six species are indicated by color density (the darker the color the more species contain the conserved region).

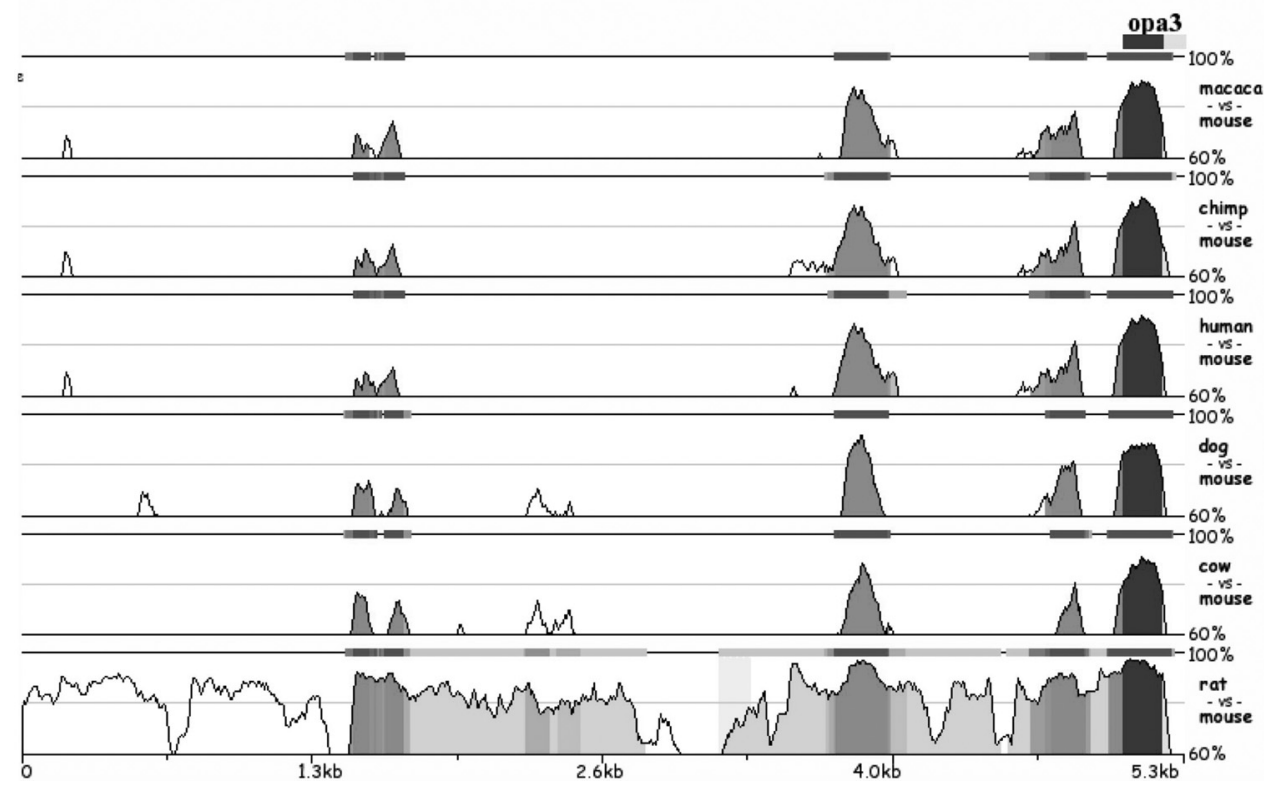

animals carrying the pL122P mutation, suggesting that Opa3 mRNA is upregulated due to the presence of the mutation. The increase in Opa3 content in mutant animals compared with wild-type littermate controls has been difficult to prove unequivocally by Western blot in lens, which may be due to accumulation and slow turnover of the protein in this tissue. Intriguingly, the mitochondrial enzyme MnSOD, which protects against oxidative damage, is similarly affected by the presence of Opa3 mutation in lens tissue. We have found that expression of MnSOD mRNA and protein are elevated in lenses of older (but are identical in young) animals carrying the mutation. We speculate that this may be a defense mechanism to protect the organism against elevated ROS production as mitochondria age. Why this might occur in the lens is not known.

Cataract is only reported in ADOA and cataract (ADOAC), caused by two dominant missense mutations reported in human OPA3. Recessive type III MGA is not associated with cataract. The mouse model reported here has a recessive phenotype and has many of the features of type III MGA. It is not

TABLE 1. Common Functional Themes in the TFBSs Found Upstream of $O p a 3$ and Which TFBSs Are Known to Play a Role in These Processes

Cellular Stress

TFBS Neuronal/Retinal Response/Apoptosis Craniofacial/Bone Adipogenesis

\begin{tabular}{|c|c|c|c|c|}
\hline P53 and P53 decamer & + & + & + & + \\
\hline CRE-BP & + & + & + & + \\
\hline AP2F & + & + & + & + \\
\hline CREB & + & + & + & + \\
\hline Stat & + & + & & + \\
\hline Oct-01 & + & + & & \\
\hline $\operatorname{Pax} 2$ & + & & + & + \\
\hline AML-1a & + & & + & \\
\hline Chx10 & + & & + & \\
\hline RBPJK & + & & & + \\
\hline Gata3 & + & & & + \\
\hline Fox01 & & + & + & + \\
\hline E2F1 & & + & + & + \\
\hline Fox04 & & + & & + \\
\hline C-EBP $a+b$ & & + & & + \\
\hline NRF 2 & & + & & + \\
\hline MZF1 & & + & & \\
\hline VDR & & & + & + \\
\hline OLF1 & & & + & + \\
\hline SP1 & & & + & + \\
\hline Pax3 & & & + & + \\
\hline ATF(inc $3,4,6$ ) & & & + & \\
\hline Cart1 & & & + & \\
\hline HFH8 & & & + & \\
\hline Gata2 & & & & + \\
\hline GR & & & & + \\
\hline
\end{tabular}
listed.

TFBS were omitted when no evidence was found of their involvement in any of the functional themes 
a model for ADOAC and does not display cataract. We do not know why humans with type III MGA do not get cataract. It has been suggested that the mutations that which give rise to ADOAC are positioned in key domains of the native protein and disrupt specific cellular functions of Opa3 that lead to the development of cataract. The mutations giving rise to type III MGA are splice site in-frame deletions and nonsense mutations. We see relative increase in Opa3 protein and cDNA/ RNA, but no cataract. This upregulation may be a physiological response to loss or reduction of function.

Since so little is known about the Opa3 protein, we used a suite of bioinformatic tools to cast further light on other possible aspects of Opa3 function and to compare the 5-kb upstream proximal sequence of the mouse opa3 gene with other homologous mammalian sequences and identify conserved TFBSs. These TFBSs cluster into functional categories related to retinal development, cellular stress response, craniofacial development, and adipogenesis (Table 1.). Some the predicted TFBSs upstream of Opa3 (Supplementary Table S1, http://www.iovs.org/lookup/suppl/doi:10.1167/iovs.10-6406/-/ DCSupplemental) are expressed in the retina. Some of those identified herein are involved in apoptotic responses to DNA damage. This association may be of significance, since Reynier et al. ${ }^{4}$ (and others) have reported that human fibroblasts carrying the $O P A 3$ c. $227 \mathrm{G}>\mathrm{A}$ (G93S) mutation were more susceptible to apoptosis induced by staurosporine than were their wild-type counterparts. Opa3 homozygous mutant mice have reduced visual acuity and display a significant loss of cells from the retinal ganglion cell/nerve fiber layer and inner nuclear layer. Another cluster of TFBSs identified in this study is involved in bone and craniofacial development. This may have significance, since $\mathrm{B} 6 ; \mathrm{C} 3-o p a 3^{\mathrm{L} 122 \mathrm{P}}$ homozygous mice are smaller than their wild-type littermates, even during embryogenesis, and they show dysmorphic craniofacial features and a misalignment of the jaw (Votruba, unpublished data, 2011). Last, some of the predicted TFBSs are involved in adipogenesis. MRI scans and necropsy of Opa3 homozygous mutant mice show a significant lack of visceral adipose tissue. Thus, the disease-related TFBS clusters identified here mirror major phenotypic features seen in the $\mathrm{B} 6 ; \mathrm{C} 3-\mathrm{Opa} 3^{\mathrm{L} 122 \mathrm{P}}$ mouse. Identification of such TFBS may provide important future insight into factors, which may regulate $O p a 3$ gene transcription, elucidating its transcriptional rules and providing important further clues to the function of the Opa3 protein.

\section{Acknowledgments}

The author thanks Mark Young (Cardiff School of Biosciences) for helpful discussions; Robert Young and Gillian Smith (Cardiff School of Optometry and Vision Sciences) for assistance with transmission electron microscopy and histology, respectively; James Colley, Ayman Hawrani, and Peter Giles (Wales Gene Park, Institute of Genetics, University Hospital of Wales, Cardiff), for sequencing and qPCR; and Fiona Mansergh (Smurfit Institute of Genetics, School of Genetics and Microbiology, Trinity College, Dublin) for assistance with lens lysates.

\section{References}

1. Alexander C, Votruba M, Pesch UE, et al. OPA1, encoding a dynamin-related GTPase, is mutated in autosomal dominant optic atrophy linked to chromosome 3q28. Nat Genet. 2000;26:211215.

2. Delettre C, Lenaers G, Griffoin JM, et al. Nuclear gene OPA1, encoding a mitochondrial dynamin-related protein, is mutated in dominant optic atrophy. Nat Genet. 2000;26:207-210.

3. Garcin RP, Raverdy S, Delthil R, et al. Sur une affection heredofamiliale associant catarate, atrophie optique, signes extra-pyramidaux et certains stigmates de la maladie de Friedreich. (Sa position nosologique par rapport au syndrome de Behr, au syndrome de
Marinesco-Sjogren et a la maladie de Friedreich avec signes oculaires.). Rev Neurol. 1961;104:373-379.

4. Reynier P, Amati-Bonneau P, Verny C, et al. OPA3 gene mutations responsible for autosomal dominant optic atrophy and cataract. J Med Genet. 2004;41:e110.

5. Ferré M, Bonneau D, Milea D, et al. Molecular screening of 980 cases of suspected hereditary optic neuropathy with a report on 77 novel OPA1 mutations. Hum Mutat. 2009;30:E692-E705.

6. Anikster Y, Kleta R, Shaag A, Gahl WA, Elpeleg O. type III 3-Methylglutaconic aciduria (Optic Atrophy Plus Syndrome, or Costeff Optic Atrophy Syndrome): identification of the OPA3 gene and its founder mutation in Iraqi Jews. Am J Hum Genet. 2001;69:12181224.

7. Costeff H, Gadoth N, Apter N, Prialnic M, Savir H. A familial syndrome of infantile optic atrophy, movement disorder, and spastic paraplegia. Neurology. 1989;39:595-597.

8. Kleta R, Skovby F, Christensen E, Rosenberg T, Gahl WA, Anikster Y. 3-Methylglutaconic aciduria type III in a non-Iraqi-Jewish kindred: clinical and molecular findings. Mol Genet Metab. 2002; 76:201-206.

9. Ho G, Walter JH, Christodoulou J. Costeff optic atrophy syndrome: new clinical case and novel molecular findings. $J$ Inberit Metab Dis. Published online November 7, 2008.

10. Huizing M, Dorward H, Ly L, et al. OPA3, mutated in 3-methylglutaconic aciduria type III, encodes two transcripts targeted primarily to mitochondria. Mol Genet Metab. 2010;100:149-154.

11. Gould S, Keller GA, Hoskenm N, Wilkinson J, Subramani S. A conserved tripeptide sorts proteins to peroxisomes. J Cell Biol. 1989;108:1657-1664.

12. Da Cruz S, Xenarios I, Langridge J, Vilbois F, Parone PA, Martinou JC. Proteomic analysis of the mouse liver inner mitochondrial membrane. J Biol Chem. 2003;278:41566-41571.

13. Ryu SW, Jeong HJ, Choi M, Karbowski M, Choi C. Optic atrophy 3 as a protein of the mitochondrial outer membrane induces mitochondrial fragmentation. Cell Mol Life Sci. 2010;67:2839-2850.

14. Chevrollier A, Guillet D, Loiseau D, et al. Hereditary optic neuropathies share a common mitochondrial coupling defect. Ann Neurol. 2008;63:794-798.

15. Pei W, Kratz LE, Bernardini I, et al. A model of Costeff syndrome reveals metabolic and protective functions of mitochondrial OPA3. Development. 2010;137:2587-2596.

16. Davies VJ, Powell KA, White KE, et al. A missense mutation in the murine Opa3 gene models human Costeff syndrome. Brain. 2008; $131: 368-380$

17. Thompson JD, Higgins GD, Gibson TJ. CLUSTAL W: improving the sensitivity of progressive multiple sequence alignment through sequence weighting, position-specific gap penalties and weight matrix choice. Nucleic Acids Res. 1994;22:4673-4680.

18. Livak KJ, Schmittgen TD. Analysis of relative gene expression data using real-time quantitative PCR and the 2(-Delta Delta c(T)) method. Methods (San Diego, Calif). 2001;25:402-408.

19. Ovcharenko I, Loots GG, Giardine BM, et al. Mulan: multiplesequence local alignment and visualization for studying function and evolution. Genome Res. 2005;15:184-194.

20. Pennacchio LA, Rubin EM. Genomic strategies to identify mammalian regulatory sequences. Nat Rev Genet. 2001;2:100-109.

21. Quandt K, Frech K, Karas H, Wingender E, Werner T. MatInd and MatInspector: new fast and versatile tools for detection of consensus matches in nucleotide sequence data. Nucleic Acids Res. 1995; 23:4878- 4884 .

22. Hubbard TJP, Aken BL, Beal K, et al. Ensembl 2007. Nucleic Acids Res. 2007;35:D610-D617.

23. Viteri G, Carrard G, Birlouez-Aragón I, Silva E, Friguet B. Agedependent protein modifications and declining proteasome activity in the human lens. Arch Biochem Biophys. 2004;427:197-203.

24. Wride MA. Proteases in the development and diseases of the lens. Expert Rev Ophthalmol (Future Drugs Network). 2007;2:833844.

25. Owczarek-Lipska M, Plattet P, Zipperle L, et al. A nonsense mutation in the optic atrophy 3 gene (OPA3) causes dilated cardiomyopathy in Red Holstein cattle. Genomics. 2011;97:51-57. 